\title{
El rol del entrenador de baloncesto en la periodización táctica de la Espoch Sede Morona Santiago Ecuador
}

\author{
DOI: https://doi.org/10.33262/ap.v3i3.1.99
}

(c) (1) (2)

\section{The role of the basketball coach in the tactical periodization of the Espoch Sede Morona Santiago Ecuador}

Danilo Ortiz Fernández. ${ }^{1}$, Alberto Alzola Tamayo. ${ }^{2} \&$ Yoel Ortiz Fernández. ${ }^{3}$

\begin{abstract}
.
The particularity of the article is to offer a work guide to basketball coaches, in which they can have a reference to the role of the coach, in the training system of tactical prioritization. This is an aspect of sports training little used at the grassroots level and in general in basketball despite having more than three decades of its introduction by the Portuguese professor Víctor Frade in his beginning essentially in football. Objective. The article aims to offer a working guide, to basketball coaches, in which they can have a reference of the role of the coach, in the training system of tactical prioritization. Methodology. Assessment of the level of relevance of the. Methodological guide for the application of tactical prioritization in basketball training, through the method of criteria of specialists This method was used to assess in the qualitative order the proposed methodological guide. The phases proposed by Cruz (2008) are assumed, the first (preliminary) allows the selection of specialists and the preparation of the questionnaire. In the second (exploration), the consultation of specialists is carried out, through the application of the rounds, to obtain reliable states of opinion. Results. An analysis of the recommendations is carried out and the improvements are determined, they are submitted to the criteria of the specialists. These determine that the changes made to the

\footnotetext{
Escuela Superior Politécnica de Chimborazo Ecuador, danilo.ortizf@espoch.edu.ec, https://orcid.org/0000-0002-4777-1495.

2 Escuela Superior Politécnica de Chimborazo Sede Morona Santiago. Ecuador. alberto.alzola@espoch.edu.ec, https://orcid.org/0000-0002-8583-2185

${ }^{3}$ Universidad de Holguín Cuba, jortiz@uho.edu.cu, https://orcid.org/0000-0001-8331-9209
} 
guide and its activities are pertinent $(11,84.61 \%$ of specialists evaluate it. Conclusion. The analysis and interpretation of the results, through the consultation of specialists, confirmed the relevance of the guide for coaches when assessing that the proposal is effective to be implemented, by contributing to the sports training of basketball athletes by contributing to better results from the educational and sports aspects of the same.

Keywords: Training, tactical periodization, guidance.

\section{Resumen}

Introducción. El artículo tiene como particularidad ofrecer una guía de trabajo, a los entrenadores de baloncesto, en la cual ellos puedan tener una referencia del rol del entrenador, en el sistema de entrenamiento de la priorización táctica. Este es un aspecto del entrenamiento deportivo poco utilizado a nivel de base y en general en el baloncesto a pesar de tener más de tres décadas de su introducción por el profesor portugués Víctor Frade en su inicio esencialmente en el futbol. Objetivo. El artículo tiene como objetivo ofrecer una guía de trabajo, a los entrenadores de baloncesto, en la cual ellos puedan tener una referencia del rol del entrenador, en el sistema de entrenamiento de la priorización táctica. Metodología. Valoración del nivel de pertinencia de la guía metodológica para la aplicación de la priorización táctica en el entrenamiento de Baloncesto, a través del método de criterio de especialistas Este método se utilizó para valorar en el orden cualitativo la guía metodológica propuesta. Se asumen las fases propuestas por Cruz (2008), la primera (preliminar) permite la selección de los especialistas y la elaboración del cuestionario. En la segunda (exploración), se realiza la consulta de los especialistas, mediante la aplicación de las rondas, para obtener estados de opinión confiables. Resultados. Se realiza un análisis de las recomendaciones y se determina las mejoras, las mismas se someten al criterio de los especialistas. Estos determinan que son pertinentes los cambios realizado a la guía y sus actividades (11, el $84.61 \%$ de los especialistas la evalúan de. Conclusión. El análisis e interpretación de los resultados, a través de la consulta de especialistas constató la pertinencia de la guía para los entrenadores al valorar que la propuesta es efectiva para ser implementada, al contribuir en el entrenamiento deportivo de los atletas de Baloncesto al contribuir a mejores resultados desde los aspectos educativos y deportivos de los mismos.

Palabras claves: Entrenamiento, periodización táctica, guía

\section{Introducción}

El artículo tiene como particularidad ofrecer una guía de trabajo, a los entrenadores de baloncesto, en la cual ellos puedan tener una referencia del rol del entrenador, en el sistema de entrenamiento de la priorización táctica. Este es un aspecto del entrenamiento deportivo poco utilizado a nivel de base y en general en el baloncesto a pesar de tener más de tres décadas de su introducción por el profesor portugués Víctor Frade en su inicio esencialmente en el futbol. Pero que por su importancia para el logro del trabajo 
educativo, táctico y técnico en el baloncesto es trascendente su extrapolación para que los entrenadores de este deporte mejoren su preparación y la de los atletas. La muestra de trabajo estuvo compuesta por 17 entrenadores, asumiendo como objetivo metodológico la aplicación de una guía de trabajo en caminada a mejorar el rol del entrenador de baloncesto, con la implementación del entrenamiento de la periodización táctica, teniendo en cuenta principios y metodologías de trabajo, lo cual permitirá mejorar la adaptación y preparación de los entrenadores al nuevo estilo de trabajo.

La preparación del deportista en la actualidad requiere de una mejor preparación de los entrenadores de Baloncestos en las diferentes categorías, los resultados a los diferentes niveles, esta superación tiene y debe ser integral en la que se impliquen diferentes áreas del saber que tributen a la maestría pedagógica de los preparadores deportivos. La propuesta tiene como objetivo la preparación integral de los entrenadores de Baloncesto para enfrentar con éxito la priorización táctica en las diferentes categorías, el diseño de investigación fue cualitativo sobre la base de la complementariedad metodológica al trabajarse con métodos cualitativos y cuantitativos, se propone una guía para los entrenadores la cual fue sometida a juicio de especialistas, los que después de dos rondas en las que se emitieron sugerencias y criterios para su mejora la validaron.

Entre los resultados estuvo que el $100 \%$ de los especialistas la evaluaron entre medianamente relevante y relevante con más del $80 \%$ para este último aspecto. Se concluyó que la guía propuesta y los cuatro elementos contentivos para su evaluación propenden a la superación de los entrenadores y por ende a un mejor logro deportivo de sus atletas.

El proceso de aprendizaje y desarrollo de los entrenadores deportivos y en especial los deportes colectivos como el baloncesto, es arduo, largo en el cual una mayoría no logra alcanzar sus metas de convertirse en grandes entrenadores, ambición a la que aspiramos casi todos los que nos decidimos por esta profesión, que nos exige niveles de conocimiento en varios campos del saber educativo, tales como Sociología, Psicología, Pedagogía y Didáctica, campos que en un inicio forman parte del perfil de nuestra formación pero que en el camino de la gran meta no todos logramos que se integren perennemente, como parte de nuestras herramientas diarias de trabajo, unos no nos actualizamos periódicamente, otros priorizamos la preparación física, técnica y olvidando que estas herramienta son la base para nuestro posterior desarrollo y crecimiento como entrenadores pues al final nuestro producto final los atletas son un ente biopsicosocial.

En la actualidad disimiles investigaciones científicas abordan al entrenador como centro de estudio, en un campo de múltiples vertientes, Para (Caña-das, Ibáñez, García, Parejo, \& Feu, 2010, como se citó en Mancha, 2018): “La organización de las competiciones se estructura en función de la edad de los jugadores. Cada período formativo del jugador precisa un tratamiento diferenciado de los contenidos, empleando para ello los medios de entrenamiento más adecuados para su desarrollo" $(\mathrm{p}, 28)$

Continuando con esta idea (Maestre, Garcés de los Fayos, Ortín y Hidalgo, 2019, como 
se citó en Mancha- Reina, Feu Ibáñez. 2020), refieren que: "el entrenador tiene la responsabilidad de proporcionar aprendizajes relacionados con la técnica y estrategia deportiva, pero también de crear situaciones psicológicas óptimas mediante las cuales los jóvenes puedan descubrir los beneficios positivos de su práctica deportiva" (p,9) (Cañadas, 2011como se citó en Gamonales año 2019) plantea, "El análisis de las tareas de entrenamiento permite conocer el posicionamiento metodológico del entrenador, así como generar nuevas teorías de entrenamiento basadas en la práctica” (p.71)

La formación con mayor titulación favorece que los entrenadores se apoyen en el conocimiento profesional de forma autodidacta, y se apoyen menos en el conocimiento que proviene des u etapa como jugador en opinión de (Feu et al. 2012, p,37).

Por su parte, Maestre, (2019) nos dice, "el entrenador tiene la responsabilidad de proporcionar aprendizajes relacionados con la técnica y estrategia deportiva, pero también de crear situaciones psicológicas óptimas mediante las cuales los jóvenes puedan descubrir los beneficios positivos de su práctica deportiva" $(\mathrm{p}, 71)$

Es por ello que los autores del artículo al tratar de definir el rol de un entrenador de baloncesto a la hora de enfrentarse al entrenamiento de la periodización táctica tomamos como sustento, el que se define como un elemento de contenido del método que define un conjunto de habilidades, competencias y responsabilidades que están relacionadas.

\section{Marco Teórico}

Ortiz F (2020) nos plantean que:

En el baloncesto actual el dominio técnico por sí solo no constituye una garantía en el desarrollo del juego es por ello que en el proceso de enseñanza y aprendizaje, la conjugación de los elementos técnicos, táctico, psicológicos, a la hora de la enseñanza, tiene que ser una herramienta básica que todo entrenador tiene que poseer, para así formar jugadores con conceptos formativos de estos tres elemento del juego, permitiendo posteriormente contar con jugadores versátiles con amplios conocimiento de la lectura del juego en las distintas situaciones que se puedan presentar, partiendo de estos elementos ir formando estilos y modelo de juego, $(\mathrm{p}, 4)$

No obstante, tenemos que señalar que el rol del entrenador que se decide por este tipo de entrenamiento que conlleva nueva forma de entender el entrenamiento causa una preparación mucho más específica, por lo que es de vital importancia tener una preparación adecuada a la hora de planificar, estructurar, entrenar y competir por tanto el rol del entrenador de baloncesto como lo entendemos.

En la ESPOCH es importante, tener un enfoque desde los principios que forma la base de esta metodología, priorización táctica, aspecto este que sea venido trabajando, que es total mente diferente a los enfoques del entrenamiento tradicionales que hoy se emplean, cuya base an sido en gran porciento provenientes de los deportes individuales. 
En este sentido. Para (Torrents, 2005, como se citó en Alzola, 2019):

El entrenamiento deportivo ha estado influenciado por la tendencia mecanicista del ser humano. A pesar que siempre se aluda necesidad de integrar todos los aspectos del entrenamiento y se propongan concepciones holísticas (integradas), la estructura sigue siendo visión cartesiana que concibe a los organismos prácticamente como maquinas construidas por diferentes partes. $(\mathrm{p}, 2)$

Forteza (2012, como se citó en Estepa M 2020) afirma sobre la planificación tradicional que la derivación de esta macro estructura a niveles de mesociclo y microciclo cae en un abismo de empirismo y tradicionalidad ortodoxa, pues las nuevas estructuras de planificación al parecer han resultado osadas y con riesgo de aplicación, lo que fuera de eufemismos vendría siendo sacrificar un ciclo olímpico. (p, 3)

Idea compartida por Sánchez (2015, como se citó en Estepa M 2020) quien menciona en la caracterización pedagógica de los entrenadores que "muchos optan por la ortodoxia en su actuar, en las relaciones humanas y en el cómo se entrena (...) no se deja nada a suerte, le gusta tener todo establecido y bajo control" (P. 16)

En resumen, Estepa M (2020) plantea.

Con ello, los entrenadores prefieren unos resultados basados en lo que ya conocen a arriesgar con otras formas de entrenamiento el rendimiento de un deportista, pues cualquier desfase en el nivel del rendimiento, estancamientoo lesión terminaría por afectar la preparación para los olímpicos que es en últimas la gran meta tanto del deportista como del entrenador, y en los cualeshay un sinnúmero de intereses. $(\mathrm{P}, 16)$

En el contexto actual los deportes de equipo como el baloncesto considerado como deporte de invasión donde su juego y accionar son muy dinámicos, sus acciones son diversa complejas y en muchos casos coticas, impredecible donde se demanda alta concentración de la atención, toma de decisiones en escasa decimas de segundos y en contextos variable y disimiles, control de los tiempo y espacios cortos es imprescindible que los entrenadores tengan un enfoque, donde se entrene y se modelen todas estas condiciones de juego desde el primer día de entrenamiento (priorización táctica)

\section{Un acercamiento a la periodización táctica}

La periodización táctica como modelo de planificación en el baloncesto, se ha utilizado poco, es un tema que escasos entrenadores tienden abordar pues es una planificación empleada en lo fundamental en el futbol en la Escuela Superior politécnica de Chimborazo se ha venido trabajando e investigando en las últimas temporadas, de ahí que se aborda un tema de suma relevancia se trata de brindar una herramienta que faciliteel trabajo de los entrenadores y más que nada orientación, una guía para los que se decidenpor utilizar la planificación de esta metodología, (la priorización táctica) que consideramos adecuada para su aplicación en el entrenamiento de baloncesto.

Tamarit, analizado por Loaiza, (2017 como se citó en Ortiz 2020) refiere que: 
La periodización táctica es una forma de organización del entrenamiento de reciente aplicación en muchos deportes colectivos. este planteamiento genuino de Vítor Frade data de finales de los años 70, pero su implantación como estructura válida no se dio hasta la consecución por el FC Oporto de José Mourinho de numerosos éxitos gracias, en parte, a esta estructuración del entrenamiento. (p. 8)

Crespo M. (2011) analiza la periodización táctica mediante una pregunta que a juicio de los autores del artículo es la clave para entender la a partir de la respuesta, y se pregunta.

¿Es el objetivo final de la periodización táctica desarrollar un jugador automático, tipo robot? La respuesta es no. De hecho, es exactamente lo contrario. La afirmación crucial de la periodización táctica es crear un "mecanismo no-mecánico" sobre la base de sólidos conceptos tácticos, para adquirir un estilo de juego eficiente "sabiendo cómo" y "sabiendo saber cómo". (p. 16)

En este sentido para, Ortiz Fernández, (2020)

Es crucial entrena en circunstancias de juego real diariamente, que la carga principal sea el trabajo táctico y todo lo demás se derive a partir de esto, que el atleta se construya secuencias de imagen a semejanza de lo que será el juego real y con ello adquirir conocimiento a través de la práctica, diaria permitiendo una mejor comprensión del juego y así poder hacer los ajustes necesarios en cada situación del juego. Permitiendo tomar las decisiones más acertadas en el menor tiempo posible. (p. 9)

Para Sánchez Buñuel (1983, como se citó en Iglesias P2007) la planificación es “el proceso mediante el cual el entrenador responsable de un proyecto mira hacia el futuro, busca y determina alternativas y vías de acción que con mayor probabilidad pueden conducir al éxito" $(\mathrm{p}, 79)$

Así mismo planificar de acuerdo con Herrera Miguel (2007, como se citó en Iglesias P 2007) "el termino planificación nos marca la acción de organizar científicamente los contenidos de entrenamiento, con la finalidad de obtener un determinado objetivo" ( $p$. 79)

Gonzale M (2011) plantea. "Planificar supone por una parte adoptar una serie de decisiones en torno al futuro que nosotros deseamos respecto a una serie de cuestiones referidas a la optimización del rendimiento deportivo"

La planificación del entrenamiento deportivo se debe entender como un proceso con una serie de decisiones que debe tomar el entrenador respecto a la mejora y la eficacia del rendimiento de un deportista en función del contexto en el que nos veamos envueltos, iniciación, niveles bajos y medios de competición o alto rendimiento deportivo. (p. 2) Por lo que, en consideración de los autores en el proceso de entrenamiento, laplanificación, 
es el componente más importante, es la base de todo el desarrollo posteriorde este proceso, demanda un caudal de conocimiento formativos específicos, además nobasta con una buena experiencia deportiva una buena formación académica sino tambiénde constantes actualizaciones de parte del instructor, entrenador, para poder acompañarlode forma adecuada con resultados buenos, que satisfagan a todos los intereseinvolucrados.

En la priorización táctica se parte de un principio base él. Supra Principio de la Especificidad.

López Cuartero platea (2019) Para que este supra principio se dé tiene que existir permanentemente una relación entre todas las dimensiones del juego y los ejercicios de entrenamiento, siendo estos específicos y representativos de nuestro Modelo de Juego, por lo que se considerará Específico cuando estén relacionados con el Modelo de Juego que se está creando (Tamarit, 2016, pág. 38; Delgado Bordonau \& Mendez Villanueva, 2012). Según Ferreira (2004, cit. por Oliveira da Silva, 2007, pág. 27) solo tiene sentido pues, durante el entrenamiento, elegir ejercicios que fomenten nuestro "jugar", creando rutinas y comportamientos tácticos fundamentales. (p. 23)

Para que exista especificidad dentro de un ejercicio, además de su relación permanente con el Modelo de Juego, deben darse estas características (Tamarit, 2016, pág. 38)

El modelo de juego no se produce de forma natural ni aparece de la nada. El modelo de juego es el núcleo central, sobre la cual se trabajan en los entrenamientos. Este modelo de juego es planteado por el entrenador y es el resultado de un fenómeno construido a partir de diferentes factores, que determinan la forma de jugar de un equipo (Tamarit, 2007, p. 33) para (Fleitas, como se citó en Alzola, et, al, 2019) "es el modelo de juego será más rico cuando más posibilite a los jugadores ampliar su propia creatividad y talento en el juego" (p. 393)

Para Martin Borrero, (2020) lo táctico está relacionada con la idea integradora de la acción motriz pero que a su vez adquiere un matiz mayor y serian denominados como aquellos medios técnicos tácticos que son colectivos, es decir con aquellas acciones motrices que dependen de la interacción de varios componentes del equipo. (p. 62)

En este sentido Ortiz Fernández (2020), entiende que:

Es crucial entrena en circunstancias de juego real diariamente, que la carga principal sea el trabajo táctico y todo lo demás se derive a partir de esto, que el atleta se construya secuencias de imagen a semejanza de lo que será el juego real y con ello adquirir conocimiento a través de la práctica, diaria permitiendo una mejor comprensión del juego y así poder hacer los ajustes necesarios en cada situación del juego. Permitiendo tomar las decisiones más acertadas en el menor tiempo posible. (p. 9)

Esto deja claro que todos los demás componentes de la preparación del deportista el entrenador tiene que adecuarla al modelo juego que va estar basado en la táctica desde el 
primer día de entrenamiento.

En el contexto actual, cada vez es más evidente la división, entre los distintos componentes de la preparación, que, en el caso de los deportes colectivo, como el baloncesto se apartan del lógico accionar de su componente principal el juego, el cual a nuestro juicio es un error pues consideramos que todo debe de estar supeditado a él por las disimiles situaciones que se dan y el poco tiempo con el que contamos para dar respuestas adecuadas.

\section{El perfil del entrenador}

Este aspecto es revisado por Feu (2012) en diferentes investigaciones realizadas por: (Ibáñez, 1996; Giménez, 2000; Pérez, 2002; Irwin, et al, 2004; Cushion, 2005; Côté, 2006; Gilbert, et al., 2006; Lemyre, et al, 2007), (Feu, Ibáñez y Gozalo, 2010 y Jiménez $\&$ Lorenzo, 2010), que refieren tres elementos fundamentales, a saber, las experiencias previas como jugador, la formación inicial y las experiencias como entrenador, a través de una práctica variada y deliberada. (p. 3), lo cual es importante tener en cuenta para poder tener una formación lo más sólida posible.

Para Mancha-Triguero (2020), Los resultados indican que, tras la armonización de la información obtenida, tanto en la literatura científica como en los grupos focales, se puede deducir que el perfil del entrenador excelente podría estar definido por tres dimensiones: disciplinar, metodológico y personal, con una posible preferencia hacia la dimensión personal. Estos resultados parecen indicar que, para que un entrenador sea considerado excelente, debe incluir en sus competencias determinadas características de la personalidad, como el equilibrio emocional, valores, liderazgo y motivación eficiente, entre otras. (p. 18)

Además, hay que entender en este engranaje, que el entrenador deportivo es clave, ya que su influencia se vea reflejada en el comportamiento competitivo, en el entrenamiento, en la conducta social y personal del deportista; por eso, cuando se tiene un adecuado proceso de enseñanza y formación en el deporte son parte de los comportamientos y conductas del entrenador.

También, se contribuye considerablemente en el desarrollo de procesos de una manera efectiva, pues su papel es relevante para que el deportista asuma la práctica de su deporte, lo que hace que se tenga interés por conocer las diferentes características y aspectos comportamentales, cognitivos y sociales de aquellos entrenadores que orientan e influencian a los deportistas, además de buenas relaciones, las cuales contribuyen en la adherencia y permanencia a todo el proceso de entrenamiento y preparación deportiva

\section{EL rol del entrenador de baloncesto}

El rol del entrenador en la actualidad ha tomado grandes dimensiones, pues con la globalización y el desarrollo tecnológico dinámico, el poder acceder a la información es cada vez más fácil, no obstante, su preparación no siempre es la más adecuada 
Para Isaza Gómez, (2019) "asumimos el deporte como un campo, desde los supuestos de Bourdieu como un espacio de fuerzas y un espacio de luchas, relacionado en caracterizar las dimensiones del campo de los programas de formación superior de entrenadores" (Bourdieu y Wacquant, 1995), (Seibel, 2004). El entrenador o formador como un sujeto que transita entre lo empírico y lo profesional en el que día a día se generan múltiples tensiones en su campo (p.112)

Muños (2011) realiza importantes apuntes en cuanto al tema al referir:

Realizar entrenamientos exclusivamente de técnica individual basados en la repetición sistemática de un ejercicio, sin que al final el jugador deba resolver situaciones reales del juego. Tenemos que proponer tareas de "transferencia", es decir, ejecutar el movimiento trabajado analíticamente (para insistir en la velocidad y precisión de movimiento) después de haber leído y decidido cual esla solución más correcta. Recordemos que nuestros jugadores juegan solos, deben relacionarse con sus compañeros, con los adversarios y con un ritmo determinado de juego. (p .3)

Los cuales se entienden de pertinentes a la hora de plantearse la planificación del trabajo en cuanto a la táctica y el trabajo en equipo.

Para Ortiz (2020). En este sentido, el entrenador debe jugar un papel activo contribuir en la comprensión de la nueva metodología ser un gestor de la misma, ser capaz de aglutinar sus deportista y colectivo técnico alrededor de esta nueva manera de entrenar. Partiendo de tres aspectos que a nuestro modo de ver son importantes.

1. Aprender Jugando.

2. Entrenar jugando

3. Desarrollo Biopsicosocial de nuestros jugadores

Por lo que es fundamental, el desarrollo físico atreves del juego, filosofía de la priorización táctica, desarrollo psicológico basado en el entrenar nuestro modelo de juego en situaciones de competición, proponiendo distintas variantes de complejidad, imponiendo a nuestros deportistas estar alertas siempre, para poder dar respuestas ante distintas situaciones de variabilidad del juego

Y apunta, Ortiz (2020) "Desarrollo social dado por la constante interrelación entre jugadores al tener que jugar aprendiendo, entrenar jugando priorizando el trabajo colectivo desde lo individual" (p. 9)

En este sentido, Para Bengoetxea B (2018) Ser un entrenador puede parecer un puesto de trabajo atractivo y que llama la atención a primera vista. Sin embargo, es una de las profesiones más duras y requieren de mucha capacitación. Las características de un buen entrenador se asemejan a las de un profesor o profesora. El éxito de los pupilos depende en gran parte del profesional al cargo y, si hay problemas, es el primero a quien se señala con el dedo. En el mundo deportivo, habitualmente, es el entrenador quien recibe la mayoría de las críticas 
cuando algo no va bien. (p. 9)

Por otra parte, el liderazgo es un concepto muy tratado en la actualidad pero que ha estado presente casi desde la creación del hombre, implícito en algunas personas con características y comportamientos especiales que componen comunidades, grupos sociales en las cuales su actuar los marca como líderes. En este particular Robbins (1996), platea que "el liderazgo es la capacidad de influenciar grupos en la consecución de metas" (p. 9)

Para Sain de Aja (1998) "La principal responsabilidad de un entrenador de baloncesto es la formación y dirección de un grupo de jugadores mediante el entrenamiento y la competición (partidos). Por lo tanto, es básico, que un entrenador sepa controlar perfectamente al grupo de jugadores, (...) (p. 4), aspectos con los cuales se coincide ya que de ello depende el éxito de todo el proceso es decir desde la enseñanza aprendizaje hasta los logros deportivos

Y enfatiza el propio autor Sain de Aja (1996) “De esta manera surgirá, espontáneamente, una "complicidad" entre el grupo y el entrenador, pero, quien, a su vez, debe dejar siempre muy claro quién es el que dirige y quien marca el camino a seguir por el grupo" (p. 4)

Cuando hablamos del liderazgo del entrenador a nivel deportivo, no debemos referirnos simplemente a las formas en que se maneja con respecto a las actividades que se desarrollan en un campo de juego. Sino que ser un buen líder representa las habilidades de ese entrenador para desenvolverse en cualquier ámbito de la vida. Tomado de https://www.acadef.es/el-liderazgo-del-entrenador/ (2021)

En la actualidad las investigaciones científicas tienen un gran porciento de consenso sobre el liderazgo que caracterizan al entrenador. Estilo persuasivo, Líder autocrático o autoritario, Líder democrático y Estilo permisivo o "Dejar hacer"

Estas clasificaciones en algunos casos son combinaciones, otras se evidencian muy fácil en el entrenador, señalar también que estos tipos de líderes van más en muchos casos con los tipos de personalidad que lo caracterizan, lo cual no significa que sea así por siempre el entrenador tiene que ser capaz de contextualizar a la hora de asumir las riendas de un equipo. Realizar un análisis exhaustivo del equipo en lo individual y colectivo en todos los aspectos de la preparación, así como su contexto social don se desenvuelve y en base a ello ver cuál de estas características del líder, encajan más y tratar de realizar los ajustes necesarios para poder responder de forma efectiva antes los retos que conllevan esta responsabilidad.

$\mathrm{Al}$ analizar desde el punto de vista de la priorización táctica, consideramos que, en el caso concreto de esta, el entrenador democrático es el que más se ajusta. Este tipo de entrenador suele ser integrador, social, participativo y cooperador. La toma de decisiones lo hace junto con sus deportistas con más experiencia. Suele ser flexible y preocupado por sus atletas, los cuales se pueden sentirse bien en el grupo, relajados. 
Tabla 1

Guía para los profesores.

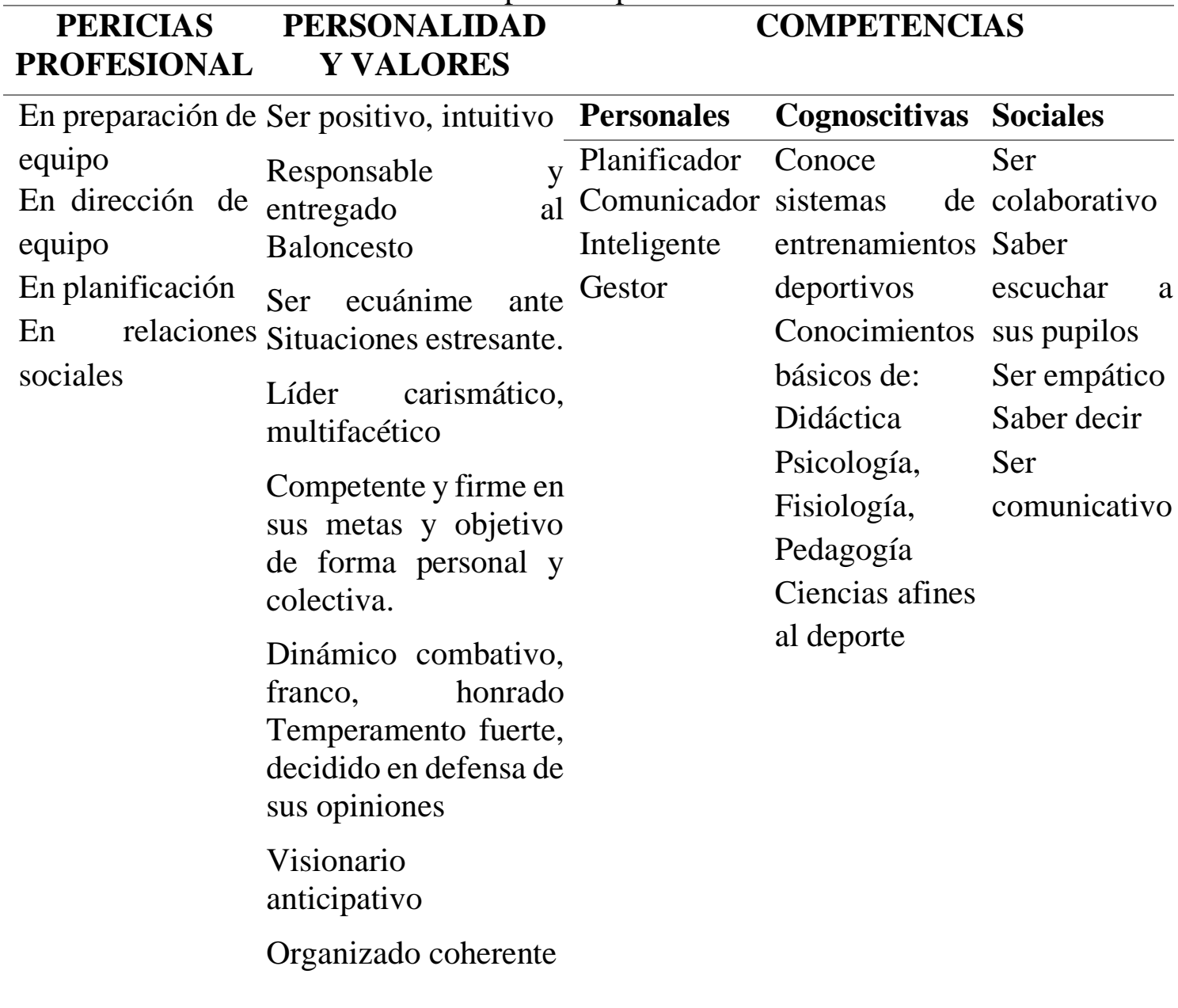

\section{Fuente de elaboración propia}

\section{Tabla 2}

Aspectos a tener en cuenta por los profesores para la priorización táctica.

\begin{tabular}{|c|c|c|c|}
\hline POSICIÓN & VISIÓN & ANTICIPACIÓN & TOMA DE DECISIÓN \\
\hline & Individual & Colectiva & \multirow{3}{*}{$\begin{array}{lll}\begin{array}{l}\text { Presagia } \\
\text { que }\end{array} & \text { lo } & \text { Preparar } \\
\text { el } & \text { entrenar jugando } \\
\text { oponente } & \text { va } & \text { en situaciónes de } \\
\text { hacer. } & & \text { competición } \\
\text { Presagia } & \text { lo } & \text { superditado o a } \\
\text { que } & \text { el la la supra- } \\
\text { oponente no } & \text { dimensión del } \\
\text { va hacer. } & \text { juego }\end{array}$} \\
\hline $\begin{array}{l}\text { Tener conciencia } \\
\text { de su } \\
\text { respetabilidad en } \\
\text { cuanto a metas y } \\
\text { objetivos } \\
\text { individuales } \\
\text { colectivos }\end{array}$ & $\begin{array}{l}\text { En la } \\
\text { captación y } \\
\text { formación } \\
\text { de talentos }\end{array}$ & $\begin{array}{l}\text { Een la formación y } \\
\text { conjugación del } \\
\text { equipo en el Modelo } \\
\text { de Juego }\end{array}$ & \\
\hline $\begin{array}{l}\text { Idea de juego. } \\
\text { De liderazgo (ser } \\
\text { líder si o si) }\end{array}$ & De sus 1 & esultados futuros & \\
\hline
\end{tabular}


Figura 1

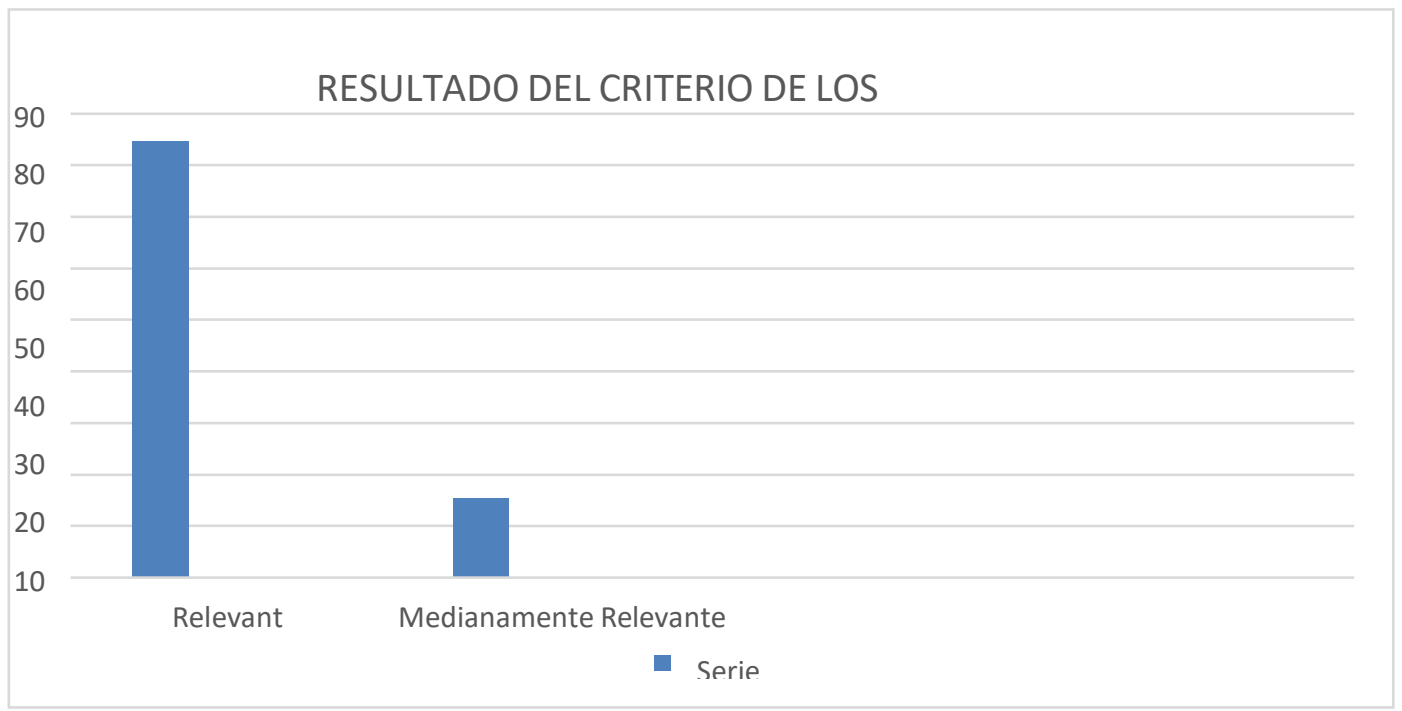

\section{Análisis de los resultados.}

Valoración del nivel de pertinencia de la guía metodológica para la aplicación de la priorización táctica en el entrenamiento de Baloncesto, a través del método de criterio de especialistas

Este método se utilizó para valorar en el orden cualitativo la guía metodológica propuesta. Se asumen las fases propuestas por Cruz (2008), la primera (preliminar) permite la selección de los especialistas y la elaboración del cuestionario. En la segunda (exploración), se realiza la consulta de los especialistas, mediante la aplicación de las rondas, para obtener estados de opinión confiables.

Esta fase se inicia con la aplicación de una encuesta a 17 profesionales todos ellos profesores de Baloncesto, profesores universitarios que se consideran posibles expertos, permitiendo determinar su coeficiente de conocimiento. Estos profesionales se desempeñan en diferentes categorías, y poseen más de 15 años de experiencia pedagógica como entrenadores. Los criterios considerados para la selección de los posibles expertos son los siguientes:

- Experiencia profesional en relación con el objeto de investigación.

- Participación en investigaciones relacionadas con la temática investigada.

- Relación de consulta de autores nacionales con la temática investigada.

- Preparación académica y científica.

- Su intuición.

En consecuencia, se seleccionaron 13 especialistas, de un total de 17 con un alto nivel de competencia y experiencia en el entrenamiento deportivo.

Fase de exploración

En esta fase se solicita a los expertos de acuerdo a las siguientes categorías evaluativa 
(Relevante, medianamente relevante, poco relevante y no relevante) los aspectos siguientes:

- La guía da respuesta a las necesidades formativas de los entrenadores y atletas desde el punto de vista de la priorización táctica a partir como un todo integrado.

- Las tres categorías declaradas (pericias profesionales, personalidad y valores y competencias) en la guía propenden a que la toma de decisiones por parte de los entrenadores es colegiada con atletas de más experiencia competitiva, de manera flexible e inclusiva.

- Considerar los cuatro aspectos a tener en cuenta por los profesores para la priorizacióntáctica que se proponen para incidir en estas.

Después de someter a evaluación por parte de los especialistas la propuesta de guía, estos consideraron, diferentes sugerencias y modificaciones las cuales se tienen en cuenta permitió perfeccionar la propuesta, su contenido y estructura, sugerencias para la mejora:

- Debe explotar más las potencialidades las relaciones sociales durante el entrenamiento como fuera de este

- Las actividades diseñas para las diferentes categorías tienen que tener uniformidad en su metodología, lo cual facilita su comprensión y descripción.

- En las competencias cognitivas debe apoyarse en el conocimiento de otras cienciasafines al entrenamiento deportivo.

Se realiza un análisis de las recomendaciones y se determina las mejoras, las mismas se someten al criterio de los especialistas. Estos determinan que son pertinentes los cambios realizado a la guía y sus actividades $(11$, el $84.61 \%$ de los especialistas la evalúan de relevante y $2,15.38 \%$ de los especialistas la evalúan de medianamente relevante) quedando aprobada la misma para su puesta en práctica con las mejoras

\section{Conclusiones:}

El análisis e interpretación de los resultados, a través de la consulta de especialistas constató la pertinencia de la guía para los entrenadores al valorar que la propuesta es efectiva para ser implementada, al contribuir en el entrenamiento deportivo de los atletas de Baloncesto al contribuir a mejores resultados desde los aspectos educativos y deportivos de los mismos

- Los profesores de Baloncesto se apropian de una herramienta que facilita una mejor preparación para la comprensión y aplicación de los fundamentos tácticos y técnicos de este deporte

- Mejora los comportamientos tácticos de los atletas en el juego de Baloncesto, lo cual favorece el logro de mejores resultados

- Aumento de la concentración de nuestros atletas en los juegos.

- Mejora en las interrelaciones personales de los atletas, al poder explotar sus habilidades individuales en función del equipo. 


\section{Bibliografia}

Alzola Tamayo, A., Ortiz Fernández, D., Vaca, M. E., \& Alzola Tamayo, S. (2019). Determinación estructural de la periodización táctica en el baloncesto femenino de la Espoch sede Morona Santiago Ecuador. Revista. Ciencia Digital, 3(3.1), 390-408. https://doi.org/10.33262/cienciadigital.v3i3.1.710.

Bengoetxea, Beñat (2018). 10 Características de un buen Entrenador https://www.esportter.com/blog/caracteristicas-de-un-buen-entrenador/

Crespo (2011). ITF Coaching and Sport Science Review; 53 (19): 16- 18

Cruz Ramírez, Miguel y Antonio Campano Peña (2008) El procesamiento de la información en las investigaciones educacionales., Editorial. Educación Cubana. La Habana 245p

Estepa Monroy (2020). Una mirada a la planificación deportiva y tendencias del entrenamiento aplicadas al Voleibol. Universidad de Ciencias Aplicadas y Ambientales UDCA Facultad de Ciencias de La Salud Ciencias del deporte Bogotá D.C.

Gamonales, J.M.; Gómez-Carmona, C.D.; Córdoba-Caro, L.G.; Ibáñez, S.J. (2019). Influencia del perfil de entrenador en el diseño de las tareas en el fútbol. Estudio de caso. Journal of Sport and Health Research. 11(Supl 1):69-82.

Isaza G. (2019). Programas de formación superior en el campo de la Educación Física, el Deporte y áreas afines en cuatro países de américa latina. Perfiles profesionales y desafíos. Revista Latinoamericana de Estudios Educativos (Colombia), vol. 15, núm. 1, Universidad de Caldas, Colombia

José M. González Ravé. (2015). La Planificación del Entrenamiento Deportivo: Cambios Vinculados a las Nuevas Formas de Entender las Estructuras Deportivas Contemporáneas Universidad de Castilla la Mancha journal Revista de Entrenamiento Deportivo, Volumen 29, Número 1.

López Cuartero (2018/2019). Periodización una propuesta práctica en fútbol base Trabajo final de grado Universidad de Castilla. Ed. La Mancha España.

M. Maestre; E.J. Garcés de los Fayos; F.J. Ortín; M.D. Hidalgo. (2019). El perfil del entrenador formativo excelente. Estudio preliminar Localización: Jornadas doctorales de la Universidad de Murcia. 2019, ISBN 978-84-09-09200-0, págs. 899-905[1] Universidad de Murcia.

Mancha Triguero (2018). ¿Afecta la fase de juego al diseño de las tareas de un equipo de baloncesto de Formación Universidad de Extremadura https://www.researchgate.ne

Mancha-Triguero, D.; Reina, M.; Feu, S. e Ibáñez, S.J. (2020) Influence of the Coach's 
Profile in Formative Basketball Training. Revista Internacional de Medicina y Ciencias de la Actividad Física y el Deporte vol. X (X) pp. xx. Http://cdeporte.rediris.es/revista.

Ortiz, F. D. (2020). Priorización táctica la toma de decisiones en la transición ofensiva en el baloncesto femenino de la ESPOCH sede Morona Santiago Ecuador. Publicado en Ciencia

Digital, https://cienciadigital.org/revistacienciadigital2/index.php/CienciaDigital/article/vi ew/13 43

Revista de Entrenamiento Deportivo (2015) La Planificación del Entrenamiento Deportivo: Cambios Vinculados a las Nuevas Formas de Entender las Estructuras Deportivas Contemporáneas Artículo publicado en el, journal Volumen 29, Número 1.,

Sebastián Feu Molina, Sergio José Ibáñez Godoy, Alberto Lorenzo Calvo. (2012). El conocimiento profesional adquirido por el entrenador de balonmano: experiencias y formación. Revista de Psicología del Deporte, vol. 21, núm. 1, pp. 107-115 Universitat de les Illes Balears Palma de Mallorca, España.

Sergio Jiménez Saiz y Maria Cañadas Alonso. (2012). Revista de Psicología del Deporte Vol. 21, núm. 1, pp. 107-115 ISSN: 1132-239X, https://cienciadigital.org/revistacienciadigital2/index.php/CienciaDigital/article/vi ew/13 43 


\section{PARA CITAR EL ARTÍCULO INDEXADO.}

Ortiz Fernández, D., Alzola Tamayo, A. ., \& Ortiz Fernández, Y. (2021). El rol del entrenador de baloncesto en la periodización táctica de la Espoch Sede Morona Santiago Ecuador. AlfaPublicaciones, 3(3.1), 325-340. https://doi.org/10.33262/ap.v3i3.1.99

\section{Ligncia}

El artículo que se publica es de exclusiva responsabilidad de los autores y no necesariamente reflejan el pensamiento de la Revista Alfa Publicaciones.

El artículo queda en propiedad de la revista y, por tanto, su publicación parcial y/o total en otro medio tiene que ser autorizado por el director de la Revista Alfa Publicaciones.
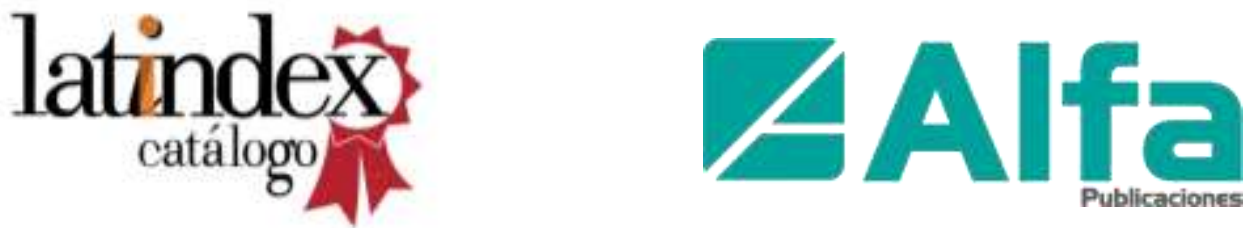